\title{
Personalized Approach of Severe Eosinophilic Asthma Patients Treated with Mepolizumab and Benralizumab
}

\author{
Laura Bergantini Miriana d'Alessandro Paolo Cameli Francesco Bianchi \\ Piersante Sestini Elena Bargagli Rosa Metella Refini \\ Department of Medical Sciences, Surgery and Neuroscience, Respiratory Disease and Lung Transplant Unit, \\ Siena University, Siena, Italy
}

\section{Keywords}

Severe asthma $\cdot$ T cells · L-selectin · Krebs von den Lungen .

Therapy · Prognosis

\begin{abstract}
Background: New anti-IL-5 antibodies, mepolizumab and benralizumab, have recently been approved for severe asthma, sharing the same inclusion criteria. Objective: To contribute on biomarkers research leading to the personalized choice, we investigated L-selectin, Krebs von den Lungen (KL-6), and lymphocyte subsets as bioindicators of airway hyper-responsiveness and remodeling. Materials and Methods: A cohort of 28 patients affected by severe eosinophilic asthma were treated with anti-IL-5 drugs. According to clinical parameters, patients were subdivided into early and partial responders. Lymphocytes subsets were analyzed through flow cytometry, while KL-6 and sL-selectin were analyzed on serum samples. Clinical, functional, and immunological data at baseline (T0), after 1 month (T1), and 6 months of therapy were collected in a database. Results: All treated patients showed an increase in the percentage of forced expiratory volume in the first second (FEV1) and FEV1/forced vital capacity ratio and a decrease of peripheral eosinophils for both drugs after 1 month of treatment. Mepolizumab-treated pa-
\end{abstract}

tients also showed decreased $\mathrm{CD}^{+}$and NKT-like cell percentages and a significant increase in SL-selectin concentrations between T0 and T1. Stratifying the cohort of our patients in early and partial responders at T0, they showed a reduction of peripheral eosinophils, sL-selectin and KL-6, while no differences were found at T0 between early and partial responders patients treated with benralizumab. Conclusions: This real-life study provides new insights for the personalized approach to severe asthma therapy. Although preliminary, the results indicate that besides eosinophils, KL-6 and sL-selectin are useful as biomarkers of early response that can also involve in the pathogenesis of severe asthma.

(c) 2020 S. Karger AG, Basel

\section{Introduction}

Severe asthma is defined as asthma requiring highdose inhaled corticosteroids (ICS and/or OCS), long-acting inhaled beta 2 agonists, and other agents (e.g., antileukotrienes) to be clinically controlled [1]. However, de-

Edited by H.-U. Simon, Bern. karger@karger.com

www.karger.com/iaa

Karger
(C) 2020 S. Karger AG, Basel

\footnotetext{
Laura Bergantini

Department of Medical Sciences, Surgery and Neurosciences Respiratory Disease and Lung Transplant Unit, Siena University Viale Bracci 1, IT-53100 Siena (Italy)
}

laurabergantini@gmail.com 
Table 1. Demographic characteristics of our cohort of severe eosinophilic patients

\begin{tabular}{lllc}
\hline & $\begin{array}{l}\text { Mepolizumab-treated } \\
\text { patients } \\
(n=20)\end{array}$ & $\begin{array}{l}\text { Benralizumab-treated } \\
\text { patients } \\
(n=8)\end{array}$ & $p$ values \\
\hline Age & $56.3 \pm 11.8$ & $50.5 \pm 16.1$ & $\mathrm{~ns}$ \\
Gender (F/M) & $13 / 7$ & $4 / 4$ & $\mathrm{~ns}$ \\
Smoking habits (former-current/never) & $12 / 8$ & $6 / 2$ & $\mathrm{~ns}$ \\
\hline
\end{tabular}

spite the clinical control of treatments and management of comorbidities, severe asthma can remain uncontrolled with increasing risks of drug-related adverse effects [2]. Most patients undergo an impairment of quality of life, and pulmonary function tests (PFTs) reveal a significant obstructive impairment of the lung function volume [3]. Although pathogenic mechanisms of these impairments are not well known, recent studies suggest of the involvement of specific endotypes (atopic, non-atopic eosinophilic, neutrophilic, paucigranulocytic, and mixed) and stimulates the research of new and therapeutic approaches $[4,5]$.

The best known pathogenic mechanism involved in severe asthma development is mediated by eosinophils and IL-5 [6]. New anti-eosinophilic monoclonal antibodies, mepolizumab and benralizumab, have recently been approved to reduce exacerbations and to improve lung function in patients with severe and uncontrolled asthma with eosinophilic inflammation [7-9]. The 2 drugs have different mechanisms of action: mepolizumab blocks the activity of circulating cytokine IL-5 and benralizumab interferes with the IL-5 receptor alpha (IL-5Ra) of eosinophils $[10,11]$. Although the role of the 2 drugs in remodeling or modulating the hyper-responsiveness of airways in severe asthma patients were partially investigated, they are very effective in reducing eosinophils in the peripheral blood, airways, and bone marrow [12-14]. In particular, mepolizumab does not abolish eosinophil peroxidase staining in the lung tissue, indicating that after mepolizumab, there are still products of eosinophil degranulation in the tissue [15].

In asthma, it has been found that L-selectin (a molecule mediating leukocyte rolling and promoting their migration into airways) plays a crucial role in the development and control of airway hyper-responsiveness. Actually, the L-selectin expression by T cells is greatly increased in asthma patients [16].

In severe asthma, the airway remodeling usually consists of abnormal changes of cells with thickness of the walls and scarred [13]. A target of remodeling and diseases activity widely investigated among fibrotic lung diseases is KL-6 [17-19]. Serum KL-6 is a mucin-like glycoprotein, over-expressed in alveolar epithelial cells, reflecting alveolar damage and regeneration of type II pneumocytes [20, 21].

As a rule, mepolizumab and benralizumab show a good safety profile, favorable clinical efficacy, and allow alternative treatment options for hyper-eosinophilic patients [22-25]. However, there are no reliable predictive markers to select patients for mepolizumab versus benralizumab (sharing the same prescription criteria) and to detect the response of each patient to treatments with these drugs [26-28].

To contribute on possible biomarker research leading to the personalized choice of the drug, we investigated a group of patients with severe eosinophilic asthma, treated with benralizumab and mepolizumab. L-selectin and KL-6 were analyzed as bioindicators of airways hyperresponsiveness and remodeling and in phenotype patients, according to their answer to the treatment.

\section{Materials and Methods}

\section{Study Design and Population}

A cohort of 28 patients (age $54.4 \pm 13.4$ years) affected by severe eosinophilic asthma were treated with anti-IL- 5 biological therapy: $20(72 \%)$ with mepolizumab (100 mg s.c. every 4 weeks) and 8 (28\%) with benralizumab (100 mg s.c. every 8 weeks). All patients were Caucasians and gave their written informed consent to participate in the study, which was approved by our Local Ethics Committee OSS-REOS (12908). There were no statistical differences in age, gender distribution, and smoking habits between the 2 groups of treated patients (Table 1), and they showed similar functional and immunological data before starting the mepolizumab and benralizumab therapy. The diagnosis of severe eosinophilic asthma, according to guidelines [1], and the monitoring of all patients were performed at the Siena University Regional Referral Centre for Rare Lung Diseases from January 2018 to March 2020. Patients were followed up for at least 6 months and stratified as (a) "partial responders" (those with a reduction in exacerbation $\geq 50 \%$, requir- 
Table 2. Functional, immunological, and serum analysis in patients at $\mathrm{T} 0$ and $\mathrm{T} 1 \mathrm{in}$ mepolizumab- and benralizumab-treated patients

\begin{tabular}{|c|c|c|c|c|c|c|}
\hline FEV1, \% & $84.4 \pm 26.6$ & $87.1 \pm 21.5$ & 0.04 & $81.3 \pm 25$ & $89.7 \pm 15$ & 0.04 \\
\hline FEV1, mL & $2,558.86 \pm 886$ & $2,820 \pm 960.7$ & 0.0037 & $2,607.5 \pm 1,067.3$ & $2,747.1 \pm 847.6$ & 0.005 \\
\hline Tiffeneau index & $69.4 \pm 12.4$ & $72.8 \pm 10.26$ & 0.04 & $66 \pm 8.4$ & $70.5 \pm 9$ & 0.04 \\
\hline $\mathrm{KL}-6, \mathrm{U} / \mathrm{mL}$ & $332.7 \pm 124$ & $310.7 \pm 107.1$ & ns & $334.6 \pm 76.8$ & $329.1 \pm 88.3$ & ns \\
\hline \multicolumn{7}{|l|}{ Lymphocytes immunophenotyping } \\
\hline $\mathrm{CD} 45$ & $19.1 \pm 5.4$ & $17.9 \pm 7.4$ & ns & $24.5 \pm 11.8$ & $28.5 \pm 1.3$ & $\mathrm{~ns}$ \\
\hline CD3 & $81.8 \pm 8.5$ & $79.5 \pm 7.7$ & ns & $81.6 \pm 5$ & $76 \pm 6.7$ & ns \\
\hline $\mathrm{CD} 4$ & $47.9 \pm 12$ & $53.5 \pm 8.4$ & ns & $58.5 \pm 3.4$ & $52 \pm 10$ & ns \\
\hline CD8 & $31.7 \pm 14$ & $24 \pm 8$ & 0.03 & $20.5 \pm 8.6$ & $22 \pm 4.8$ & ns \\
\hline
\end{tabular}

ing maintenance with oral corticosteroids [mOCS] or a $\geq 50 \%$ reduction in prednisolone dose); (b) "early responders" (patients without exacerbation and off mOCS at 6 months of follow-up) [29]. All patients tolerated the therapy without any major side effects and clinical, functional, and immunological data at baseline (T0), after 1 month (T1), and 6 months of therapy (T6 the latter only for mepolizumab treated patients) were collected in a database.

\section{Pulmonary Function Tests}

PFTs were performed according to the ATS/ERS standard parameters [30] using the Jaeger body plethysmograph with corrections for temperature and barometric pressure. Forced expiratory volume in the first second (FEV1) and FEV1/forced vital capacity (FVC) index were expressed as $\mathrm{ml}$ and percentage of predicted values, respectively, and FEV1/FVC index was expressed as percentages.

\section{Preparation and Storage of PBMCs}

Cytofluorimetric analysis was performed at baseline and after 1 month of therapy for all patients. Blood collection tubes were available at 6 months for mepolizumab-treated patients. Experiments were performed at Siena University in the respiratory diseases lab, Respiratory Diseases and Lung Transplant Unit, from January 2019 to March 2020. Lymphocyte subset percentages in PBMCs from these patients were determined by flow cytometric analysis. The peripheral blood samples were collected after 8-h fasting in a tube containing EDTA anticoagulants (BD Vacutain$\mathrm{er}^{\circledR}$ EDTA Tubes, BD Biosciences, San Jose, CA, USA) and processed within $8 \mathrm{~h}$. Briefly, a layer of blood was added to $15-\mathrm{mL}$ Ficoll Histopaque ${ }^{\circledR}-1077$ (Sigma-Aldrich, Inc.) St. Louis, MO, USA) in a conical 50-mL tube and centrifuged for $30 \mathrm{~min}$ at 3,000 $\mathrm{rpm}$ in a swinging-bucket rotor without brake. The mononuclear cell layer was transferred to a new conical 50-mL tube (Corning ${ }^{\circledR}$ $50 \mathrm{~mL}$ centrifuge tubes, Sigma-Aldrich, Inc.), adding $15 \mathrm{~mL}$ RPMI 1640 medium (Gibco ${ }^{\circledR}$ - Thermo Fisher Scientific, Inc., Waltham,
MA, USA), and centrifuged at 1,500 rpm for $10 \mathrm{~min}$. Supernatant was carefully removed, and the cells were stored in liquid nitrogen till the end of the experiments.

\section{T Lymphocyte Detection by Flow Cytometry}

Blood samples were processed by flow cytometry using a panel of monoclonal antibodies (BD Multitest ${ }^{\mathrm{TM}}$ 6-color TBNK, San Jose, CA, USA), including FITC-labeled CD3, PE-labeled CD16 and CD56, PerCP-Cy5.5-labeled CD45, PE-Cy7-labeled CD4, APC-labeled CD19, and APC-Cy7-labeled CD8. Briefly, a total of $10^{6}$ cells were stained with $20 \mu \mathrm{L}$ of antibody cocktail for $30 \mathrm{~min}$. Washing was performed with $1 \mathrm{~mL}$ RPMI 1640, followed by centrifuging for $10 \mathrm{~min}$ at $1,500 \mathrm{rpm}$. The supernatant was discarded and the cell pellet resuspended in $400 \mu \mathrm{L}$ RPMI medium. At least 100,000 events were read by flow cytometer for each sample. Data were analyzed using DIVA software (BD Biosciences, San Jose, CA, USA). Lymphocytes were distinguished on the basis of forward versus side scatters, and additional gating was applied using side scatters versus CD45 to distinguish lymphocytes from cell debris. Specific panels were subsequently assessed to identify T lymphocytes, B lymphocytes, and NK cells. T-lymphocyte subpopulations were gated in order to distinguish $\mathrm{CD}^{+} \mathrm{CD}^{+}$(T-helper), $\mathrm{CD}^{+} \mathrm{CD}^{+}$(T-cytotoxic), and $\mathrm{CD} 3^{+} \mathrm{CD} 16 / 56^{+}$(NKT) cells.

\section{Serum Soluble L-Selectin Detection Analysis}

Blood samples obtained before starting the therapy and after 1 month of follow-up were collected for all patients until the analysis were done. Serum soluble L-selectin quantification was performed by bead-based multiplex LEGENDplex ${ }^{\mathrm{TM}}$ analysis (LEGENDplex $^{\mathrm{TM}}$ Adhesion Molecule Panel [Biolegend]) according to the manufacturer's instructions. Reactions were run in duplicate. The minimum detectable concentration is $88.7 \pm 47.9 \mathrm{pg} / \mathrm{mL}$. Analysis was performed with BD FACSCanto II flow cytometer (BD Biosciences, San Jose, CA, USA). Data were processed by LEGENDplex V8.0 software (Biolegend), and concentrations were expressed in $\mathrm{ng} / \mathrm{mL}$. 


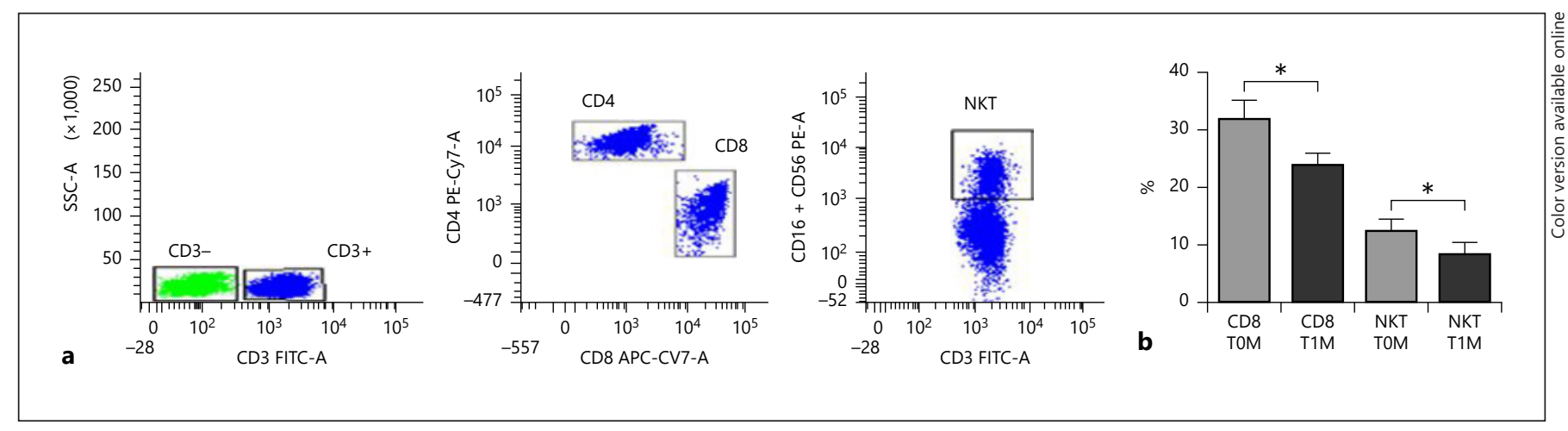

Fig. 1. a Flow cytometry strategy for detection of $\mathrm{CD}^{+}, \mathrm{CD}^{+}, \mathrm{CD} 8^{+}$, and NKT-LIKE cells. CD3 is gated on $\mathrm{CD}_{4} 5^{+}$. b Different percentages of CD8 and NKT cells in mepolizumab-treated patients at baseline (T0) and after 1 month of therapy (T1).

Krebs von Den Lungen-6 Assay

Krebs von den Lungen- 6 was measured in serum by KL-6 reagent assay (Fujirebio Europe, UK) as previously reported [31]. The principle of the assay is agglutination of sialylated carbohydrate antigen in samples with KL- 6 mAb by antigen-antibody reaction. The change in absorbance reflects KL- 6 concentrations. The detection limit of the assay is $13.47 \mathrm{U} / \mathrm{mL}$ consistent with the guidelines in the CLSI Protocol EP17-A. KL-6 concentrations in samples were expressed in $\mathrm{IU} / \mathrm{mL}$.

\section{Statistical Analysis}

The results (expressed as mean \pm SD or median and interquartile range) were not normally distributed, and the $\chi^{2}$ test was used for categorical variables. The Mann-Whitney $U$ test comparison was used for comparative analysis between mepolizumab- and benralizumab-treated patients and between "early responders" and "partial responders" patients treated with mepolizumab. We also performed the logistic regression in order to verify the response to mepolizumab therapy. In the logistic regression, early responses were tested as dependent variable, while KL-6, peripheral eosinophils, and sL-selectin were tested as independent variables. We also assessed the validity of variables used to distinguish early and partial responders by areas under curves (AUCs) in the receiver operating characteristic (ROC). Sensitivity, specificity, and positive and negative predictive values were calculated for cutoffs of the different variables. The Youden index $(J=\max$ [sensitivity + specificity -1$]$ ) was used to establish the best cutoffs for the prediction of response to treatment.

The statistical significance $(p<0.05)$ of differences between different times of treatments was assessed with the Wilcoxon matched-pairs signed rank test. Statistical analysis and graphic representation were performed by GraphPad Prism 8.0 software.

\section{Results}

\section{Functional and Immunological Parameters}

Table 2 reports functional and immunological parameters, including peripheral blood eosinophils and KL-6 and sL-selectin concentrations at time 0 and time 1 in benralizumab and mepolizumab populations. FEV1 (in $\mathrm{mL}$ and \%) and FEV1/FVC ratio significantly increased after 1 month of mepolizumab or benralizumab therapy associated with a significant decrease of peripheral eosinophilia (in cells $/ \mathrm{mm}^{3}$ and \%).

Mepolizumab-treated patients also showed significant decrease of $\mathrm{CD}^{+}$and NKT-like cells percentages between $\mathrm{T} 0$ and $\mathrm{T} 1$ and a concomitant significant increase in L-selectin concentrations (Fig. 1).

After 6 months of mepolizumab treatment (T6), PFTs, immunological data, KL-6 concentrations, and number of eosinophils were available for 14 patients. The results showed significant decrease of peripheral eosinophils and significant increase of FEV1/FVC ratio and FEV1 (\%). The peripheral percentages of NKT-like cells resulted in a significantly decrease after 6 months of mepolizumab treatment, as reported in online suppl. Table 1 (see www. karger.com/doi/10.1159/000508936 for all online suppl. material).

\section{Metapolizumab Treatment: "Early Responders" versus} "Partial Responders"

Stratifying the cohort of our patients in "early responders" $(n=10)$ and "partial responders" $(n=10)$, at T0 they showed significant differences in peripheral eosinophils, sL-selectin, and KL-6 (Fig. 2). At T1, the "partial responders" group showed significant variations in peripheral eosinophilia, sL-selectin concentrations, and percentages of NK cells, and the "early responders" showed significantly different values of peripheral eosinophilia, sL-selectin concentrations, percentages of NKT-like cells, KL-6, FEV1, and FEV1/FVC ratio. 


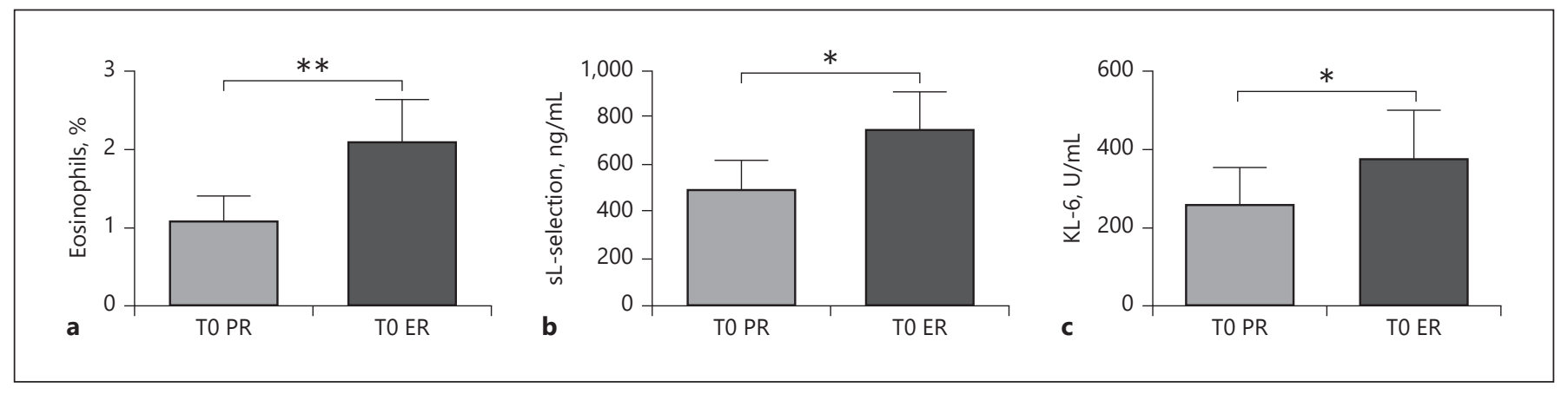

Fig. 2. a Differences of eosinophils (\%) at baseline (T0) in mepolizumab-treated patient subgroups: ER and PR. b Differences of sL-selectin $(\mathrm{ng} / \mathrm{mL})$ at baseline (T0) in mepolizumab-treated patient subgroups: ER and PR. c Differences of KL-6 (U/mL) at baseline (T0) in mepolizumab-treated patient subgroups: ER and PR. T0PR, baseline values of mepolizumab: PR subgroup; T0ER, baseline values of mepolizumab: ER subgroup. ${ }^{* *} p<0.001$, ${ }^{*} p<0.05$. ER, early responders; PR, partial responders.

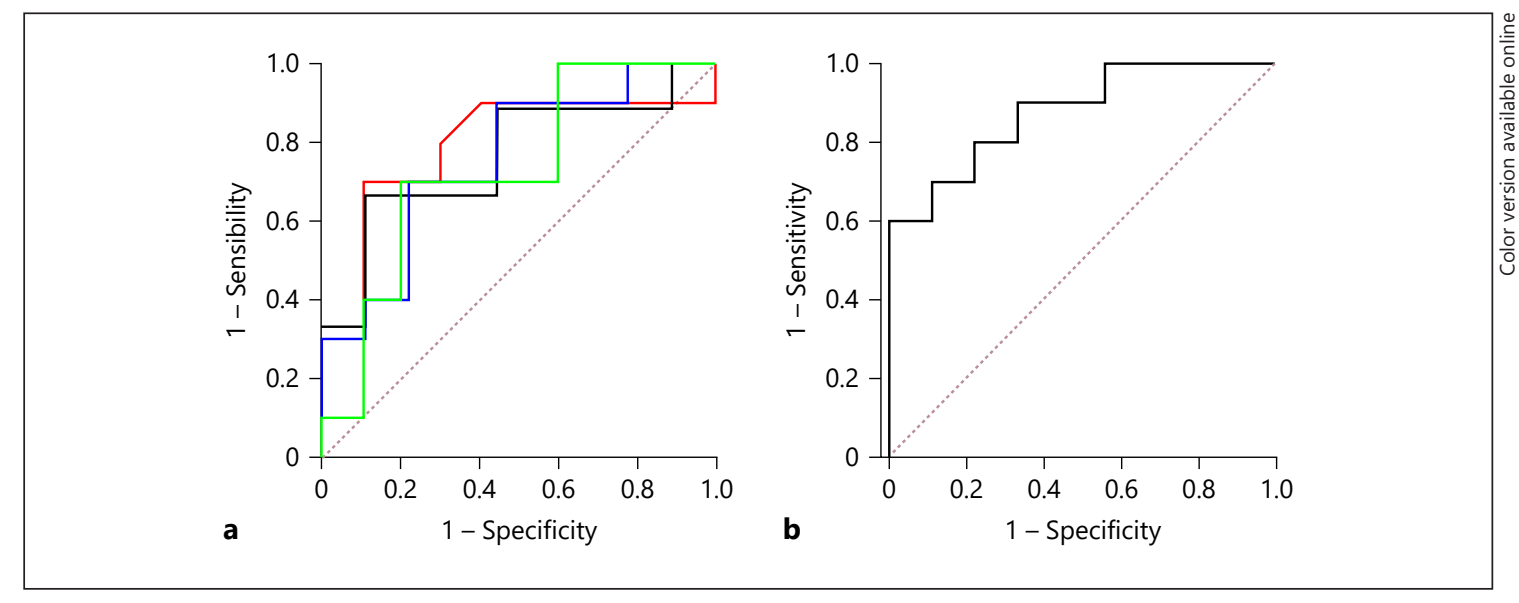

Fig. 3. a ROC curve analysis of KL-6 (blue), eosinophils (red), Tiffeneau index (black), and sL-selectin (Green) in discriminating early responders from partial responders in the mepolizumab group. $\mathbf{b}$ ROC curve analysis of logistic regression model: eosinophils, KL-6, and sL-selectin. ROC, receiver operating characteristic.

Benralizumab Treatment: "Early Responders" versus "Partial Responders"

No differences were found at T0 between 5 "early responders" and 3 "partial responders" patients treated with Benralizumab. At T1, the 2 groups showed statistically significant changes in peripheral eosinophilia (676 \pm 365 cell $\mathrm{mm}^{-3}$ vs. 0 cell mm $\mathrm{mm}^{-3} ; p<0.0001,8.5 \pm 4.1$ vs. $0 \% ; p<0.0001$ in "partial responders," and $760 \pm 679$ cell $\mathrm{mm}^{-3}$ vs. 0 cell mm ${ }^{-3} ; p<0.0001,8 \pm 5.6$ vs. $0 \% ; p<0.0001$ in "early responders").

\section{Predictor Biomarkers of Patients' Responses to Mepolizumab Treatment}

Patients treated with mepolizumab therapy were stratified according to the clinical response, and ROC analysis was performed to identify baseline cutoff values of our proposed biomarkers to distinguish "early responders" from "partial responders" (Fig. 3a). The results showed better AUCs for "early responders" than for "partial responders": for KL-6, AUC = 0.75, 95\% CI: 0.53-0.97, $p=$ 0.004; peripheral eosinophils, AUC $=0.79,95 \%$ CI: $0.57-$ $1, p=0.04$; FEV $1 / \mathrm{FVC}$ ratio, $\mathrm{AUC}=0.76,95 \% \mathrm{CI}: 0.53-$ $0.99, p=0.005$; and sL-selectin, AUC $=0.73,95 \% \mathrm{CI}$ : $0.50-0.95, p=0.003$. In the logistic regression, the early response to mepolizumab therapy was tested as dependent variable, while KL-6, peripheral eosinophils, and Lselectin were tested as independent variables.

The combination of these biomarkers allowed an improved identification of "early responders." The ROC curve analysis of the model revealed an AUC of 0.8778 (95\% CI: 
$0.72-1$; negative predictive value (\%): 77.78 , positive predictive value (\%): 80; $p=0.005$ ) (Fig. 3b). A cutoff value of KL-6 more than $337 \mathrm{IU} / \mathrm{mL}$ had $70 \%$ sensitivity and $77.78 \%$ specificity; peripheral eosinophilia values higher than a cutoff of $580\left(\right.$ cells $/ \mathrm{mm}^{3}$ ) showed $80 \%$ sensitivity and $70 \%$ specificity. Finally, sL-selectin concentration higher than a cutoff of 1,051 (ng/mL) showed 70\% sensitivity and $80 \%$ specificity facilitating the discrimination between "early responders" and "partial responders" patients.

\section{Discussion}

This survey on 28 patients with severe eosinophilic asthma confirmed the safety and efficacy of the treatment with the new anti-IL-5 and anti-IL-5Ra monoclonal antibodies mepolizumab and benralizumab. In agreement with literature data after a month of therapy, we recorded the improvement of their clinical status and functional parameters with significant increases of FEV1 and FEV1/ FVC ratio values and a decrease of peripheral eosinophilia $[23,24,32-34]$. The role of eosinophils in severe asthma and the capability of mepolizumab and benralizumab to markedly reduce blood eosinophils are well known. However, as previously reported, we found that the benralizumab treatment leads to the complete depletion of eosinophils [34-37]. Interestingly, the mepolizumab treatment changed values of sL-selectin, $\mathrm{CD}^{+}$, and NKTlike cells after the first dose.

$\mathrm{CD} 8^{+} \mathrm{T}$ cells are an important source of type 2 cytokines. They are insensitive to corticosteroids, and the induction of asthma exacerbations is often mediated via corticosteroid-resistant pathways [36]. NKT-like cells are mainly reported in the lungs of severe poorly controlled asthma and play a potential role in airway hyper-reactivity, together with other Th2 cells or independently of adaptive immunity responses [37]. Hodge et al. [38] suggested in poorly controlled asthma an altered expression of cytotoxic/pro-inflammatory mediators by different lymphocyte subsets such as $\mathrm{CD}^{+} \mathrm{NK}$ and NKT-Like cells. This immunological pattern of expression was proposed as a marker of treatment responsiveness and/or risk of exacerbations [38]. In the context of NK and NKTlike cells, Duvall et al. [39] pointed out that NK cells are altered in severe asthma.

In line with these data, our results reported an altered expression of $\mathrm{CD}^{+}$and NKT-like cells after mepolizum$\mathrm{ab}$ than benralizumab treatment. This difference, worth of further investigation, may be related to the differential molecular target of these 2 treatments.

Biomarkers of Severe Eosinophilic Asthma
The exact role of circulating adhesion molecules, including sL-selectin is still controversial. However, we found significant changes in sL-selectin concentrations after the first month of mepolizumab therapy. According to Hamzaoui et al. [40], in severe asthma patients, circulating soluble forms of these molecules may reflect different inflammatory pathways and could be used to monitor the disease activity.

The stratification of our patient population showed that higher levels of peripheral eosinophils, sL-selectin, and KL-6 concentrations at baseline were associated with early response to mepolizumab treatment. Combination of these 3 blood biomarkers significantly improved the identification of "early responders" patients. Comparisons between baseline values and those at T1 showed that peripheral eosinophils and sL-selectin changed significantly in all patients, and in "early responders," there was a significant increase of FEV1 and FEV1/FVC ratio and a decrease of KL- 6 and NKT-like cells concentrations. In the benralizumab cohort, after 1 month of therapy, the only difference between "early and partial responders" were eosinophils values. Unfortunately, the small size of the cohort did not allow a better evaluation of this aspect.

Although blood eosinophils and severe asthma features are considered the best predictor biomarkers for anti-IL-5 and anti-IL-5Ra therapies, the detection of blood eosinophils has great limitation and does not support the clinicians in choosing the more suitable drug [38]. For the first time, this study identified sL-selectin as a marker of hyper-responsiveness and KL-6 as an indicator of airway remodeling, suggesting a new panel of blood biomarkers discriminating "early responders" from "partial responders" among severe eosinophilic asthma patients.

\section{Conclusion}

By stratifying a cohort of 28 severe eosinophilic asthma patients treated with 2 new anti-IL- 5 and anti-IL-5Ra monoclonal antibodies (mepolizumab and benralizumab), this study provides new insights for the personalized approach to severe asthma therapy. Although preliminary, the results indicate that besides peripheral eosinophils (nowadays considered the best predictor biomarkers for anti-IL-5 and anti-IL-5R $\alpha$ therapies), KL-6 and sLselectin are useful novel biomarkers of early response to mepolizumab (interfering with pathogenesis of severe asthma).

Int Arch Allergy Immunol 2020;181:746-753 DOI: $10.1159 / 000508936$ 


\section{Statement of Ethics}

All subjects gave their written informed consent to the study. The study was approved by local ethical committee OSS-REOS (12908).

\section{Disclosure Statement}

The authors have no conflicts of interest to declare.

\section{Funding Sources}

The present work was performed at Siena University and there are no funding sponsors to declare.

\section{Author Contributions}

L.B.: conceived the study and supervised all aspects of study; L.B. and M.d.: data analysis; P.C. and F.B.: interpretation of results, collection of data, and built database; and P.S. and E.B.: drafted the paper.

\section{References}

1 Chung KF, Wenzel SE, Brozek JL, Bush A, Castro M, Sterk PJ, et al. International ERS/ ATS guidelines on definition, evaluation and treatment of severe asthma. Eur Respir J. 2014 Feb;43(2):343-73.

2 Song WJ, Lee JH, Kang Y, Joung WJ, Chung KF. Future risks in patients with severe asthma. Allergy Asthma Immunol Res. 2019 Jul 8; 11(6):763-78

3 Boskabady MH, Rezaeitalab F, Rahimi N, Dehnavi D. Improvement in symptoms and pulmonary function of asthmatic patients due to their treatment according to the Global Strategy for Asthma Management (GINA). BMC Pulm Med. 2008 Dec 22;8:26.

4 Carr TF, Zeki AA, Kraft M. Eosinophilic and non-eosinophilic asthma. Am J Respir Crit Care Med. 2018 Jan 1;197(1):22-37.

5 Agache I. Non-eosinophilic asthma endotypes. Curr Treat Options Allergy. 2015 Sep 1; 2(3):257-67.

6 Yilmaz I. Selection of biologics for severe type-2 asthma. J Investig Allergol Clin Immunol. 2019;29(5):399-400

7 Bleecker ER, Wechsler ME, FitzGerald JM, Menzies-Gow A, Wu Y, Hirsch I, et al. Baseline patient factors impact on the clinical efficacy of benralizumab for severe asthma. Eur Respir J. 2018;52(4):1800936.

8 Poulakos MN, Cargill SM, Waineo MF, Wolford AL. Mepolizumab for the treatment of severe eosinophilic asthma. Am J Health Syst Pharm. 2017 Jul 1;74(13):963-9.

9 Dávila González I, Moreno Benítez F, Quirce S. Benralizumab: a new approach for the treatment of severe eosinophilic asthma. J Investig Allergol Clin Immunol. 2019 Apr; 29(2):84-93.

10 Bagnasco D, Caminati M, Ferrando M, Aloè T, Testino E, Canonica GW, et al. Anti-IL-5 and IL-5Ra: efficacy and safety of new therapeutic strategies in severe uncontrolled asthma. BioMed Res Int. 2018;2018:5698212.

11 Simon HU, Yousefi S, Germic N, Arnold IC, Haczku A, Karaulov AV, et al. The cellular functions of eosinophils: Collegium Internationale Allergologicum (CIA) Update 2020. Int Arch Allergy Immunol. 2020;181(1):11-23.
12 Chapman DG, Irvin CG. Mechanisms of airway hyper-responsiveness in asthma: the past, present and yet to come. Clin Exp Allergy. 2015 Apr;45(4):706-19.

13 Fehrenbach H, Wagner C, Wegmann M. Airway remodeling in asthma: what really matters. Cell Tissue Res. 2017;367(3):551-69.

14 Bergantini L, Cameli P, d'Alessandro M, Vietri L, Perruzza M, Pieroni M, et al. Regulatory $\mathrm{T}$ cells in severe persistent asthma in the era of monoclonal antibodies target therapies. Inflammation. 2020 Apr;43(2):393-400.

15 Kelly EA, Esnault S, Liu LY, Evans MD, Johansson MW, Mathur S, et al. Mepolizumab attenuates airway eosinophil numbers, but not their functional phenotype in asthma. Am J Respir Crit Care Med. 2017 01;196(11): 1385-95.

16 Nadi E, Hajilooi M, Pajouhan S, Haidari M. Soluble L-selectin as an independent biomarker of bronchial asthma. J Clin Lab Anal. 2015 May;29(3):191-7.

17 d'Alessandro M, et al. Krebs von den Lungen- 6 as biomarker for disease severity assessment in interstitial lung disease: a comprehensive review. Biomark Med. 2020. 10.2217/ bmm-2019-0545.

18 d'Alessandro M. Serum KL-6 concentrations as a novel biomarker of severe COVID-19. Med Virol. 2020. 10.1002/jmv.26087.

19 d'Alessandro M, Bergantini L, Cameli P, Lanzarone N, Antonietta Mazzei M, Alonzi V, et al. Serum KL-6 levels in pulmonary Langerhans' cell histiocytosis. Eur J Clin Invest. 2020 Apr 20;e13242.

20 Bergantini L, Bianchi F, Cameli P, Mazzei MA, Fui A, Sestini P, et al. Prognostic biomarkers of sarcoidosis: a comparative study of serum chitotriosidase, ACE, lysozyme, and KL-6. Dis Markers. 2019;2019:8565423.

21 Bergantini L, Bargagli E, Cameli P, Cekorja B, Lanzarone N, Pianigiani L, et al. Serial KL-6 analysis in patients with idiopathic pulmonary fibrosis treated with nintedanib. Respir Investig. 2019 May;57(3):290-1.
22 Kurosawa M, Sutoh E. Prospective open-label study of 48-week subcutaneous administration of mepolizumab in japanese patients with severe eosinophilic asthma. J Investig Allergol Clin Immunol. 2019;29(1):40-5.

23 Pertzov B, Unterman A, Shtraichman O, Shitenberg D, Rosengarten D, Kramer MR. Efficacy and safety of mepolizumab in a realworld cohort of patients with severe eosinophilic asthma. J Asthma. 2019 03;1-6.

24 Liu T, Wang F, Wang G, Mao H. Efficacy and safety of benralizumab in patients with eosinophilic asthma: a meta-analysis of randomized placebo-controlled trials. Front Med. 2018 Jun;12(3):340-9.

25 Cameli P, et al. A Comprehensive Evaluation of Mepolizumab Effectiveness in a Real-Life Setting. Int Arch Allergy Immunol. 2020. 10.1159/000507996.

26 Yancey SW, Keene ON, Albers FC, Ortega H, Bates S, Bleecker ER, et al. Biomarkers for severe eosinophilic asthma. J Allergy Clin Immunol. 2017 Dec 1;140(6):1509-18.

27 Karaulov AV, Garib V, Garib F, Valenta R. Protein Biomarkers in Asthma. Int Arch Allergy Immunol. 2018;175(4):189-208.

28 Tomasiak-Lozowska MM, Zietkowski Z, Przeslaw K, Tomasiak M, Skiepko R, BodzentaLukaszyk A. Inflammatory markers and acidbase equilibrium in exhaled breath condensate of stable and unstable asthma patients. Int Arch Allergy Immunol. 2012;159(2):1219.

29 Kavanagh JE, d'Ancona G, Elstad M, Green L, Fernandes M, Roxas C, et al. Real-World effectiveness and the characteristics of a "superresponder" to mepolizumab in severe eosinophilic asthma. Chest. 2020 Apr 8;S00123692(20):30574-2.

30 Culver BH, Graham BL, Coates AL, Wanger J, Berry CE, Clarke PK, et al. Recommendations for a standardized pulmonary function report. An official American thoracic society technical statement. Am J Respir Crit Care Med. 2017 01;196(11):1463-72. 
31 Lanzarone N, Gentili F, Alonzi V, Bergantini L, d'Alessandro M, Rottoli P, et al. Bronchoalveolar lavage and serum KL- 6 concentrations in chronic hypersensitivity pneumonitis: correlations with radiological and immunological features. Intern Emerg Med. 2020 Feb 20.

32 Ortega HG, Liu MC, Pavord ID, Brusselle GG, FitzGerald JM, Chetta A, et al. Mepolizumab treatment in patients with severe eosinophilic asthma. N Engl J Med. 2014 Sep 25;371(13): 1198-207.

33 Farne HA, Wilson A, Powell C, Bax L, Milan SJ. Anti-IL5 therapies for asthma. Cochrane Database Syst Rev. 2017 21;9:CD010834.
34 Nair P, Wenzel S, Rabe KF, Bourdin A, Lugogo NL, Kuna P, et al. Oral glucocorticoidsparing effect of benralizumab in severe asthma. N Engl J Med. 2017 22;376(25):2448-58.

35 Busse W, Chupp G, Nagase H, Albers FC, Doyle S, Shen Q, et al. Anti-IL-5 treatments in patients with severe asthma by blood eosinophil thresholds: Indirect treatment comparison. J Allergy Clin Immunol. 2019;143(1): 190-200.e20.

36 Hinks TSC, Hoyle RD, Gelfand EW. CD8+ Tc2 cells: underappreciated contributors to severe asthma. Eur Respir Rev Off J Eur Respir Soc. 2019 Dec 31;28(154):190092.

37 Umetsu DT, Dekruyff RH. Natural killer T cells are important in the pathogenesis of asthma: the many pathways to asthma. J Allergy Clin Immunol. 2010 May;125(5):975-9.
38 Hodge S, Hodge G, Simpson JL, Yang IA, Upham J, James A, et al. Blood cytotoxic/inflammatory mediators in non-eosinophilic asthma. Clin Exp Allergy. 2016 Jan;46(1):60-70.

39 Duvall MG, Barnig C, Cernadas M, Ricklefs I, Krishnamoorthy N, Grossman NL, et al. Natural killer cell-mediated inflammation resolution is disabled in severe asthma. Sci Immunol. 2017 Mar 10;2(9):eaam5446. https:// www.ncbi.nlm.nih.gov/pmc/articles/ PMC5561743/.http://dx.doi.org/10.1126/sciimmunol.aam5446

40 Hamzaoui A, Ammar J, El Mekki F, Borgi O, Ghrairi H, Ben Brahim M, et al. Elevation of serum soluble E-selectin and VCAM-1 in severe asthma. Mediators Inflamm. $2001 \mathrm{Dec}$; 10(6):339-42. 